\title{
Fast Monte Carlo Method in Stochastic Modelling of Charged Particle Multiplication
}

\author{
Alla V. Shymanska*, Vitali A. Babakov \\ School of Computer and Mathematical Sciences, Auckland University of Technology, Private Bag 92006, \\ Auckland 1142, New Zealand. \\ * Corresponding author. Email: alla.shymanska@aut.ac.nz \\ Manuscript submitted January 26, 2015; accepted June 4, 2015. \\ doi: 10.17706/ijapm.2015.5.3.218-226
}

\begin{abstract}
Development of electronic devices, where the secondary electron emission is used to amplify input signals, requires simulation of complicated stochastic processes. The essence of the method proposed here consists of separating the amplification process into serial and parallel stages. The mean and variance of the amplitude distribution at the output of the entire system are obtained using the mean and variance of each stage. Theorems about serial amplification stages and parallel amplification paths constitute the key part of the method with partial Monte Carlo simulation for one simple stage. Here, the method is used to investigate the effect of the contact conducting layer on the gain and the noise factor of the channel amplifier. The case of image converters and intensifiers with an inverting electron-optical system and microchannel plate as an amplifier is taken for the consideration.
\end{abstract}

Key words: Contact conducting layer, microchannel electron amplifier, Monte Carlo simulations, noise factor, theorems about serial, parallel amplification stages.

\section{Introduction}

Amplification by secondary electron emission has many advantages and makes it possible to detect even single photons. If electrons or other particles strike the solid surface, secondary electrons are emitted from it whose number can be much higher than the number of primary particles. According to the form, in which the successive amplification of current is being obtained, we can distinguish photomultipliers and channel multipliers.

The principle of a photomultiplier is shown in Fig. 1. Electrons emitted from the photocathode are focused by the input focusing system and reach the first dynode. The material of the dynode has a secondary emission yield (SEY) $\sigma>1$, so that the number of electrons starting from the first dynode is $\sigma$ times greater. These electrons are focused to the next dynode, and the process is repeated so that the number of electrons rises as an avalanche.

Channel multipliers have similar to the photomultipliers principals of multiplication but have no discrete multiplication stages (Fig. 2). The whole multiplier consists of cylindrical dynode having a small diameter. The accelerating voltage is applied to the ends of this cylinder. If the inner surface has SEY $\sigma>1$, the electron escaping from the surface at the entrance of the channel will provoke an avalanche developing in the direction towards the end of the channel. Channel multipliers are developed as single devices (channel trons) and as microchannel plates (MCP), which is an array of single parallel channels (Fig. 2). They are widely used for the detection and amplification of electrons, ions and neutrons, as well as UV and $X$-ray 
photons. They are very attractive for use in particle physics, astronomy, in medical X-ray imaging, image converters and intensifiers etc.

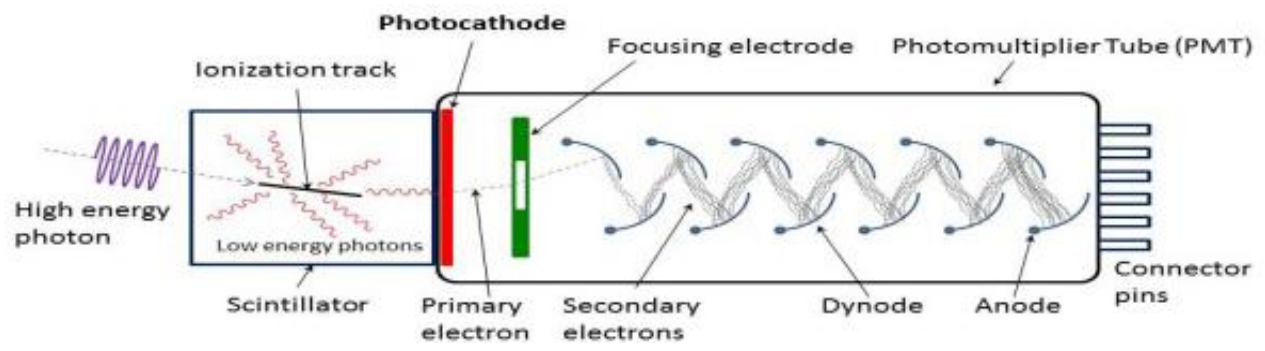

Fig. 1. Photomultiplier.

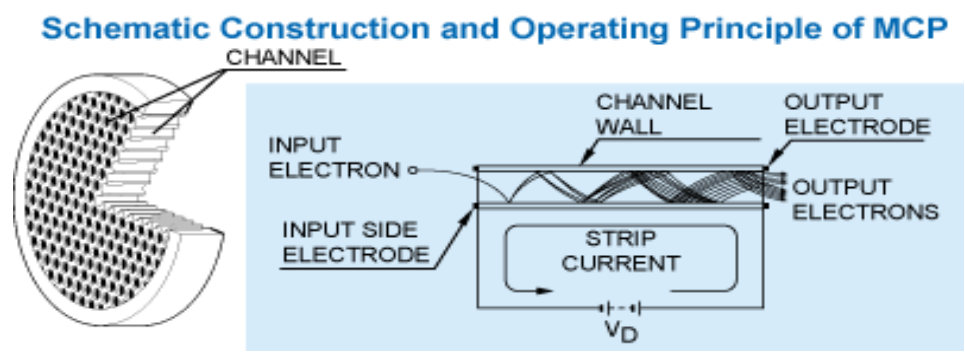

Fig. 2. Channel amplifier and MCP.

Both types of amplifiers cause an increase of noise and therefore a deterioration of the signal-to-noise ratio. Despite a number of remarkable properties, poor noise characteristics are the main drawback of systems with channel amplification. Particularly, MCP is the main noise source of low-level light image converters and intensifiers.

Development of such devices require simulation of complicated stochastic processes of the secondary electron emission. It is usually done by the use of Monte Carlo (MC) methods [1]-[6]. However, the direct simulation of the entire amplification process by the MC methods requires considerable computer time. Optimization of a device's parameters in terms of gain, noise and quality of image makes the simulation especially difficult or even impossible. Moreover, it is difficult to evaluate a contribution of different system's parameters to the entire amplification process, and their effect on output characteristics.

The essence of the method proposed here consists of separating the amplification process into serial and parallel stages. The mean and variance of the amplitude distribution at the output of the entire system are obtained using the mean and variance of each stage. Theorems about serial amplification stages and parallel amplification paths [7] constitute the key part of the method. Combination of the theorems and MC simulations, which are used once for one simple stage, enables one to conduct complicated investigations and optimizations. The method preserves all advantages of the MC simulations: represents real physical processes fully and adequately, and uses experimental characteristics completely in the model. Moreover, splitting a stochastic process into a number of different stages, allows a contribution of each stage to the entire process to be easily investigated. The method provides high calculation accuracy with minimum expenditures of the computer time.

Here, the method is used to investigate the effect of the contact conducting layer on the gain and the noise factor of the channel amplifier. The case of image converters and intensifiers with an inverting electron-optical system (EOS) and MCP as an amplifier is taken for consideration.

\section{The Theorems}




\subsection{Theorem about Serial Amplification Stages}

The mean and variance of the entire multiplication process can be calculated using the mean and variance of the separate sequential stages. Let $p_{k}(v)$ be the probability distribution of the number of particles at the output of the $k$-th stage, produced by one particle at its input. Let $m_{k}$ be the mean and $d_{k}$ be the variance of the $p_{k}(v)$. Then the generating function of the probability distribution $p_{k}(v)$ is

$$
q_{k}(u)=\sum_{v=0}^{\infty} u^{v} p_{k}(v), \text { where }|u| \leq 1
$$

Based on the approach used in[8] we can construct the generating function for the probability distribution of the number of particles after the last $(N$-th) stage:

$$
\boldsymbol{Q}_{N}(\boldsymbol{u})=\boldsymbol{q}_{\mathbf{0}}\left(\boldsymbol{q}_{\mathbf{1}}\left(\boldsymbol{q}_{2}\left(\ldots\left(\boldsymbol{q}_{N}(\boldsymbol{u})\right) \ldots\right)\right)\right)
$$

To find the mean $M$, and variance $D$ of the amplitude distribution $P_{N}(v)$ after the $N$-th stage, we convert the expressions (1) to the logarithmic generating functions, introducing new variables:

$$
v=\ln u h_{k}(v)=\ln \sum_{v=0}^{\infty} e^{v v} p_{k}(v) H_{N}(v)=\ln \sum_{v=0}^{\infty} e^{v v} P(v)
$$

Then the expressions (1) can be written as $H_{N}(v)=h_{0}\left(h_{1}\left(h_{2}\left(\ldots\left(h_{N}(v)\right) \ldots\right)\right)\right)$, where $H_{N}(v)$ is the logarithmic generating function of the distribution $P_{N}(v) ; h_{k}(v)$ is the logarithmic generating function of the distribution $p_{k}(v)$.

Differentiating $H_{N}(v)$ with respect to $v$ once and using the propertiesof the logarithmic generating functions, with $v=0$ we obtain the mean value of $P_{N}(v)$ :

$$
M=m_{0} m_{1} \ldots m_{k} \ldots m_{N}=\prod_{k=0}^{N} m_{k}
$$

Differentiating $H_{N}(v)$ with respect to $v$ twice, with $\boldsymbol{v}=0$ we obtain the variance $D$ after the $N$-th stage of this multistep sequential process.

$$
\begin{aligned}
D=d_{0}\left(m_{1} m_{2} \ldots\right. & \left.m_{N}\right)^{2}+d_{1} m_{0}\left(m_{2} m_{3} \ldots m_{N}\right)^{2}+d_{2} m_{0} m_{1}\left(m_{3} m_{4} \ldots m_{N}\right)^{2} \\
& +\cdots+d_{k} m_{0} m_{1} \ldots m_{k-1}\left(m_{k+1} m_{k+2} \ldots m_{N}\right)^{2}+d_{N} m_{0} m_{1} \ldots m_{N-1}
\end{aligned}
$$

or:

$$
D=\sum_{k=0}^{N} d_{k} \prod_{i=0}^{k-1} m_{i} \prod_{j=k+1}^{N} m_{j}^{2}
$$

The expressions (2) and (3)constitute the theorem of serial amplification stages [7].

\subsection{Theorem of Parallel Amplification Paths}

The mean and variance of the amplitude distribution at the output of the system with some parallel amplification paths can be calculated using the mean and variance of each path. Let the primary particle be multiplied along one of $n$ possible parallel paths, and $\rho_{k}$ be the probability of choosing the $k$-th path. If each path gives an average of $g_{k}$ particles at the output with a variance of $v_{k}$, then the mean $G$ and the variance $V$ of this multiplication process can be obtained. 
Let $\varphi_{k}(v)$ be the probability distribution of the numberof particles $v$ at the output of the $k$-th path produced by one particle at its input. Then the probability distribution $\Phi(v)$ of the number of particles at the output of the entiresystem of $n$ parallel paths will be $\Phi(v)=\sum_{k=1}^{n} \rho_{k} \varphi_{k}(v)$. Then the mean $G$ is:

$$
G=\sum_{v=0}^{\infty} \Phi(v) v=\sum_{k=1}^{n} \rho_{k} \sum_{v=0}^{\infty} \varphi_{k}(v) v=\sum_{k=1}^{n} \rho_{k} g_{k}
$$

The variance $V$ of the distribution at the output of the system can be written as

$$
V=\sum_{v=0}^{\infty} \Phi(v) v^{2}-\left[\sum_{v=0}^{\infty} \Phi(v) v\right]^{2}
$$

where the first sum can be transformed to

$$
\sum_{v=0}^{\infty} \Phi(v) v^{2}=\sum_{k=1}^{n} \rho_{k} v_{k}+\sum_{k=1}^{n} \rho_{k} g_{k}^{2}
$$

Taking into account that $\left[\sum_{v=0}^{\infty} \Phi(v) v\right]^{2}=G^{2}$ the final expression is:

$$
V=\sum_{k=1}^{n} \rho_{k} v_{k}+\sum_{k=1}^{n} \rho_{k} g_{k}^{2}-G^{2}
$$

where $G$ is determined by (4). Equations (4) and (5) constitute the theorem of parallel amplification paths [7] and can be used for discrete and for continuous systems, where sums should be replaced by integrals.

\section{Electron Multiplication in Channel Amplifier}

\subsection{Physical Picture}

The theorems above with combination of partial MC simulation enable one to optimize the channel amplifier in terms of the noise factor, which is a measure of the loss of available information. Particularly, the effect of the contact conducting layer on the noise factor is under investigation. The following real physical picture is considered in the modeling. Electrons of a primary parallel monochromatic beam hit the walls of the channel at different incidence coordinates and angles (Fig. 3). Different incident angles increase the divergence of SEY after the first collision of the primary electrons. Due to the spread of the incident coordinates, the lengths of the channel, along which the multiplication of primary electrons occur, are different.

After the first collision each primary electron produces secondary electrons with different emission energy and directions. The secondary electrons are multiplied until they leave the channel. When all electrons have emerged from the channel, the yield of the individual pulse is known.

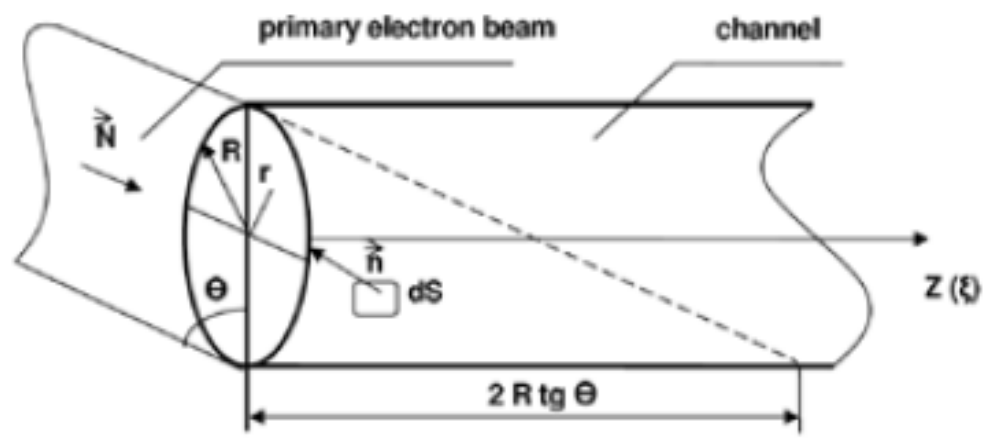

Fig. 3. Input of the primary electron beam. 
The gain of individual pulses is fluctuated considerably, and the pulse amplitude distribution changes from Poisson distribution at the beginning of the channel to a negative exponential form at its output. The length of the channel, where the amplitude distribution changes and stabilizes, is determined in [7] and called "the effective channel length". The mean gain and the variance of the amplitude distribution at the output of a system define the noise factor of an amplifier, which is greater for the negative exponential distribution than for the peaked one.

In this work the case of image converters and intensifiers with an inverting EOS and MCP as an amplifier is taken for consideration. The MCP is placed behind the anode diaphragm close to the screen [9]. To accelerate the secondary electrons toward the exit of the channel, the voltage is applied by depositing a contact conducting layer at its ends. The electrostatic field of the EOS, the field inside the channel and contact conducting layer create a nonuniform electrostatic field at the entrance of the channel. Since there is no potential drop along the conducting layer, the conditions for the movement of secondary electrons in this region are different from motion of electrons in a uniform field inside the channel.

Existing models, based only on full MC simulations, neglect the effect of the nonuniform field and the spread in incident coordinates of the primary electron beam, but all these factors are taken into account in the computational model, proposed here.

\subsection{Computational Model}

A computational model for simulation of stochastic processes of an electron multiplication in channel amplifiers is based on 3D MC simulations, and theorems about serial amplification stages and parallel amplification paths. The entire multiplication process in the channel amplifier is split into a number of sequential stages and parallel multiplication paths. Multiplication of a single electron, emitted at the beginning of the channel, is considered as a separate stage. It is simulated by 3D MC methods in the homogeneous field along the "effective channel length" which is equal half of the channel [7]. The functions $g(z)$, the mean, and $d(z)$, the variance ( $z$ is the coordinate directed along the channel axis and measured from its beginning), are calculated for $0 \leq z \leq L / 2$, where $L$ is the coordinate of the end of the channel.

The process of MC simulations uses a random number generating procedure [4], [7] to sample the various distributions such as: the distribution of the actual yield of secondaries after each collision, the emission energy, and the direction of each secondary electron.

For $n$ electrons, leaving the first half of the channel, the incidence coordinates $\xi_{k}>L / 2$ and SEY $\sigma_{k}$ are determined. The amplification in the second half of the channel is considered to consist of $n$ parallel paths. Each path has two sequential stages: first collision and multiplication of a single electron until it leaves the channel. The functions $g(z)$ and $d(z)$ along the entire channel length $(0 \leq z \leq L)$ are calculated using expressions (2)-(5).The functions $g(z)$ and $d(z)$,and the theorems allow us to conduct further investigations and optimizations without any additional MC simulations, what significantly reduces the cost of computations, and provides highly accurate results.

The part of the channel with nonuniform field is considered as a separate stage. If the initial conditions of the collision of the primary electrons with the channel walls are changed (incident angle or energy, length of the conducting layer or a high-efficiency emitter etc.), MC simulations are conducted only in the region of nonuniform electrostatic field. Then functions $g(z)$ and $d(z)$ are used to find the mean and variance of the distribution at the output of the channel, using the theorems. The nonuniform field at the entrance of the channel is calculated by solving the Laplace's partial differential equation in cylindrical coordinate system. The trajectories of the electrons in the nonuniform field are calculated by the Runge-Kutta method.

Fig. 4 shows the nonuniform field at the entrance of the channel. Computational results have been obtained for the channel diameter $d=10 \mu \mathrm{m}$, the voltage on the channel $U=800 \mathrm{~V}$, and the sputtering depth of the contact layer at the channel input $h=0.5 d$. 


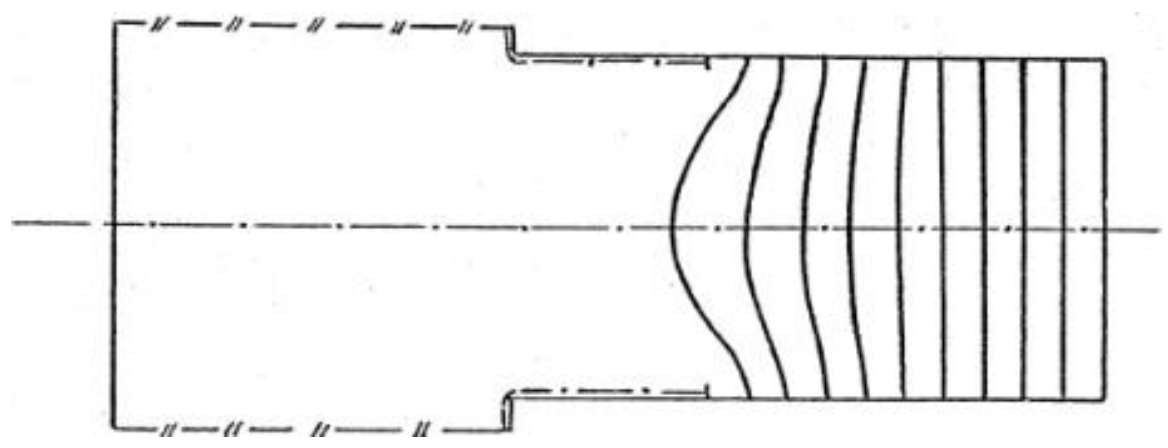

Fig. 4. Nonuniform field at the entrance of the channel.

The portion of the channel from an elementary area at its input, where the collision occurred, to the output of the channel (Fig. 3) can be considered as the amplification path when the spread in incidence coordinates of the input electrons was taken into account [10]. In such case, the variance $D$ and the average gain $G$ at the output of the multiplier can be defined using (4) and (5), where the sums should be replaced by integrals over the surface of the channels, bombarded by the electrons of the primary beam:

$$
G=\int_{s} \psi(s) g(s) d s, D=\int_{s} \psi(s) d(s) d s+\int_{s} \psi(s) g^{2}(s) d s-G^{2},
$$

where $\psi$ is the probability density for the particle to strike the elementary surface $d s ; g(s)$ is the average number of particles with variance $d(s)$ at the output of the path.

Using the theorems, finally we obtain [10]:

$$
\begin{gathered}
G=\frac{2}{\pi R} \cot \theta \int_{0}^{\pi / 2} \sigma(\varphi, V, \theta) \cos \varphi d \varphi \int_{0}^{2 R \tan \theta \cos \varphi} g(z, L) d z \\
D=\frac{2}{\pi R} \cot \theta \int_{0}^{\frac{\pi}{2}} \sigma(\varphi, V, \theta) \cos \varphi d \varphi \int_{0}^{2 R \tan \theta \cos \varphi}\left[g^{2}(z, L)+d(z, L]\right) d z+ \\
\frac{2}{\pi R} \cot \theta \int_{0}^{\pi / 2} \sigma^{2}(\varphi, V, \theta) \cos \varphi d \varphi \int_{0}^{2 R \tan \theta \cos \varphi} g^{2}(z, L) d z-G^{2},
\end{gathered}
$$

where $\varphi$ is the azimuthal coordinate in the cylindrical system; $z=2 R \tan \theta \cos \varphi$ is the border of the area of the cylindrical channel, bombarded by the electrons of the primary beam (Fig. 3).

The noise factor $F$ can be defined as $[4,7]$ :

$$
F=\gamma^{-1}\left(1+\frac{D}{G^{2}}\right)
$$

where $G$ and $D$ are defined by (6) and (7).

\subsection{Computational Results}

The computational results, obtained here, reflect the influence of the nonuniform electrostatic field and the spread of the incident coordinates of the primary electrons on the gain and the noise factor. The area covered by the input electron beam depends on the direction angle $\theta$ (Fig. 3).

At small angles $\theta$ the collision of the primary electrons with the channel wall occurs near the channel edge in the region of the week electrostatic field. The secondary electrons in this region are not accelerated 
sufficiently to continue the multiplication process. As a result, the gain is less than in the case of the uniform field at the entrance of the channel. Moreover, a considerable part of the secondary electrons with a negative velocity does not turn back because of the absence of the field, and leaves the channel.

At large angles $\theta$ the large spread in the collision coordinates of the input electrons with the channel walls increases the variance of the amplitude distribution at the output of the channel. When the penetration depth of the input electrons is increasing the number of multiplication stages is decreasing, and the gain is decreasing as well. For the angle $\theta$, where the effects, described above, are in balance, a minimum noise factor is observed, and such angle $\theta$ can be considered as an optimum for the given coverage depth.

In order to increase gain and reduce the noise factor, a layer with increased SEY is deposited on the top of the contact conducting layer at the entrance of channels. However, due to the effect of nonuniform electrostatic field and spread in incidence coordinates of the input electron beam, the noise factor can even increase [11].

Fig. 5 shows the dependence of the mean gain $g(z, L)$ on the emission coordinate $z$ of a secondary electron exiting the high efficiency emitter of the length $h=5 d$, deposited on the top of the contact conducting layer. Curve 1 has been calculated for the uniform field at the entrance of the channel, curve 2 is relevant to nonuniform field formed by the contact conducting layer (what is the practical case). When the high efficiency emitter is deposited on the top of a contact conducting layer (curve 2) only electrons, emitted from the border of the high efficiency emitter (here, it is $z>4 d$ ), take part in multiplication. It is seen that the loss of secondary electrons, emitted in the area of "week" field, is significant.

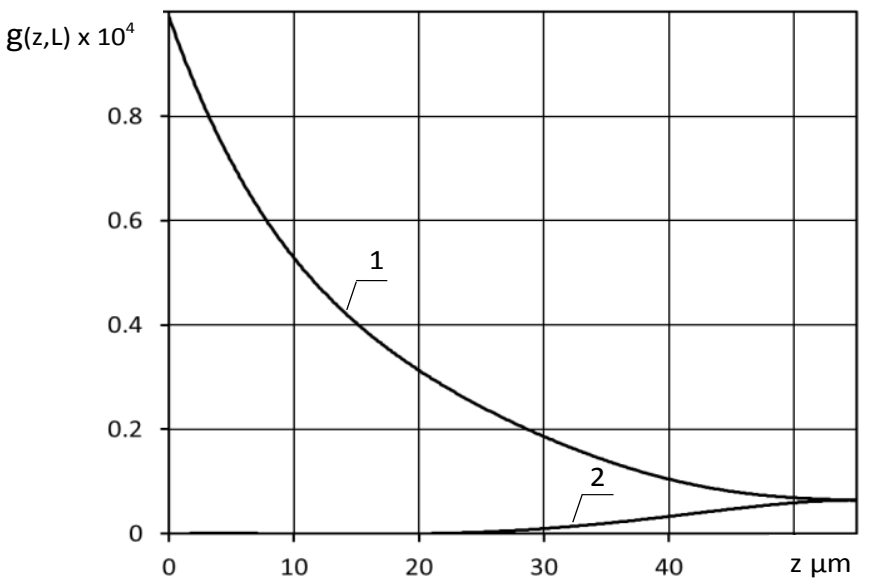

Fig. 5. Mean gain g as function of emission coordinatez (1 - uniform field; 2 - nonuniform field).

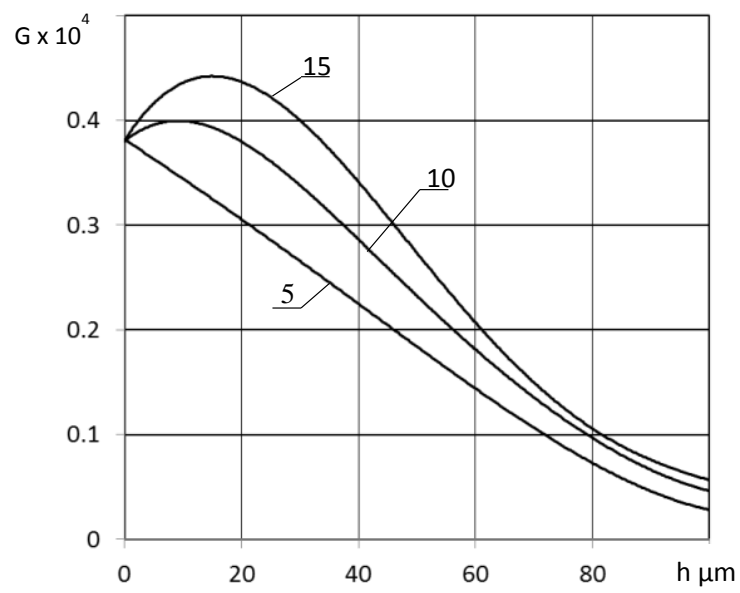

Fig. 6. Dependence of gain $G$ on length $h$ of contact conducting layer for different SEY. 
Fig. 6 and Fig. 7 show dependence of the gain $G$ and the noise factor $F$ at the output on the length of the contact conducting layer $h$ for different values of SEY. It is seen that the gain is decreasing and the noise is increasing regardless of the value of SEY.

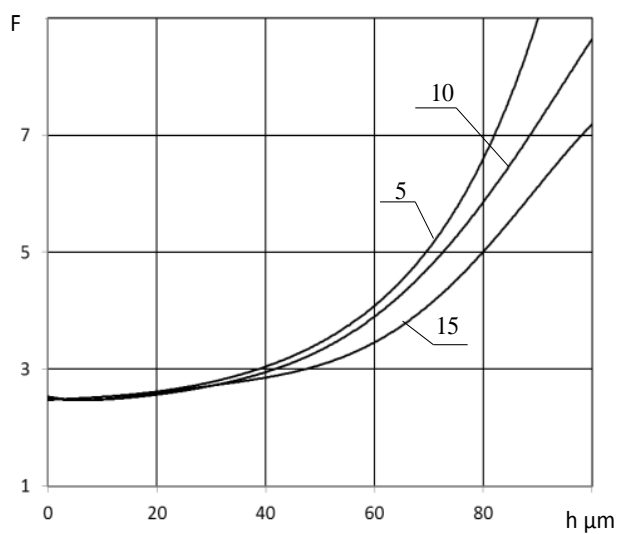

Fig. 7. Dependence of noise F on length $h$ of contact conducting layer for different SEY.

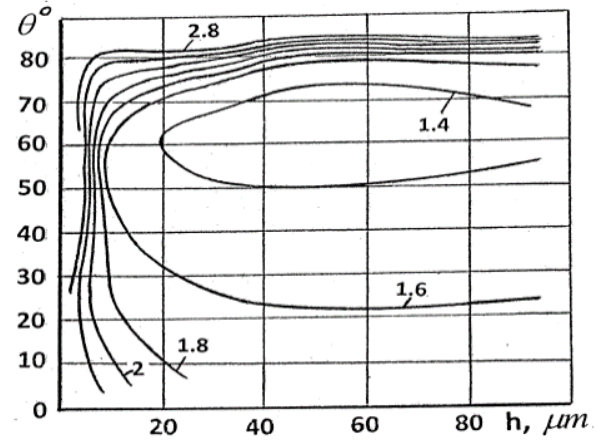

Fig. 8. Dependence of noise factor on incidence angle $\theta$ and length $h$ of high-efficiency emitter.

The summary results are shown on the Fig. 8 as the function $F(\theta, h)$, where $\theta$ is the incidence angle of the primary electron beam, and $h$ is the length of the high-efficiency emitter deposited on the top of the contact conducting layer. The energy of the primary electron beam was $V=1 \mathrm{kV}$. These results make it possible to determine the optimum combination of the parameters, providing the minimal noise factor and the region where it is stable. Thus, here $\theta \simeq 70$ and $h \bumpeq 5 d$ are optimum values, which provide the minimum noise factor $F=1.4$. It must be pointed out that the range of parameter values $50<\theta<75$ and $h \backsim 2 d$ - $5 d$ ensures the smallest value of the noise factor which practically does not depend on variations in $\theta$ and $h$.

\section{References}

[1] Binkley, D. M. (1994). Optimization of scintillation-detector timing system using Mote Carlo analysis. IEEE Trans. Nucl. Sci., 41, 386-393.

[2] Choi, Y. S., \& Kim, J. M. (2000). Monte Carlo simulations for tilted - channel electron multipliers. IEEE Trans. Electron. Devices, 47, 1293-1296.

[3] Giudicotti, L. (2002). Analytical, steady-state model of gain saturation in channel electron multipliers. Nucl. Instr. and Meth. A, 480, 670-679.

[4] Guest, A. J. (1971). A computer model of channel multiplier plate performance. Acta Electron, 14, 79-97.

[5] Price, G. J., \& Fraser, G. W. (2001). Calculation of the output charge cloud from a microchannel plate. Nucl. Instr. and Meth. A, 474, 188-196. 
[6] Shikhaliev, P. M., Ducote, J. L., Xu, T., \& Molloi, S. (2005). Quantum efficiency of the MCP detector: Monte Carlo Calculation. IEEE Trans. Nucl. Sci., 52, 1257-1262.

[7] Shymanska, A. V. (2010). Computational modeling of stochastic processes in electron amplifiers. J. Comput. Electron, 9, 93-102.

[8] Yanoshi, L. (1955). Statistical problems of an electron multiplier. Zh. Eksp. Teor.Fiz., 9, 679-696.

[9] Shymanska, A. V. (2011). Numerical analysis of electron optical system with microchannel plate. J. Comput. Electron, 10, 291-299.

[10] Shymanska, A. V., \& Babakov, V. A. (2014). Computational models for investigation of channel amplifier's optimal parameters. J. Comput. Electron., 13,161-169.

[11] Shymanska, A. V. (2015). Effect of high efficiency emitter on noise characteristics of electron amplifiers. J. Comput. Electron.

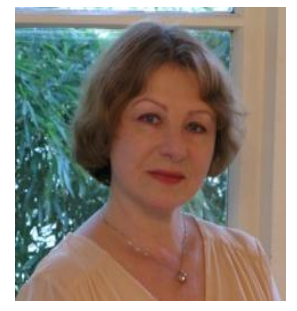

Alla Shymanska has her BEng in electronics and $\mathrm{PhD}$ in mathematics and physics (St Petersburg). She has a long research and teaching experience in areas of physical electronics and charged particle optics at different research centers and universities in Russia and Ukraine. Currently she continues her research and teaches range of papers in mathematics and computer science at School of Computer and Mathematical Sciences of Auckland University of Technology, Auckland, New Zealand.

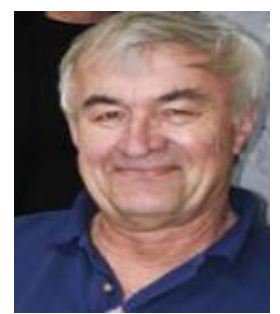

Vitali Babakov has his $\mathrm{PhD}$ and doctor of science degrees in mathematics (Novosibirsk). His major research areas are mathematics and newtonian mechanics. For many years he teaches calculus for engineering students and statistics for business students at Auckland University of Technology (New Zealand). His narrow field of activity is theory elasticity and plasticity, where V. Babakov investigates mathematical problems that can be applied for practical needs. 